\title{
MADRES E HIJOS \\ DOS ETAPAS EVOLUTIVAS BENEFICIADAS POR LA INCLUSIÓN UNIVERSITARIA
}

\author{
María Julieta D’Avirro \\ UNPAZ (Universidad Nacional de José C. Paz) \\ julietadavirro@hotmail.com \\ https://doi.org/10.17060/ijodaep.2017.n1.v2.938
}

Fecha de Recepción: 8 Marzo 2017

Fecha de Admisión: 1 Abril 2017

\section{RESUMEN}

El proyecto que se presenta indaga las representaciones sociales (RRSS) sobre la maternidad y el rol de alumno universitario, en alumnas - madres de la Universidad Nacional de José C. Paz, provincia de Buenos Aires, Argentina. El objetivo del estudio es analizar la influencia de tales RRSS en la permanencia de las alumnas madres en el ámbito universitario. Los métodos para la recolección de datos son: la entrevista semi-dirigida y la asociación libre de palabras. Se espera que los datos obtenidos acerca del grupo estudiado sean útiles para diseñar estrategias de inclusión en función de su perfil de especial vulnerabilidad. La inclusión educativa es definida por la UNESCO como el proceso de identificar y responder a la diversidad de las necesidades de todo estudiante, reduciendo la exclusión del sistema educativo. Pensar en la plena inclusión universitaria de las mujeres madres conlleva modificaciones en las estructuras determinantes del dispositivo universitario, cambios de estrategias y de estructuras.

Las representaciones sociales exploradas en la investigación, permitieron identificar no sólo necesidades del alumnado, que impactan de modo crucial en su inserción académica, sino también el impacto de su inclusión universitaria en sus estilos de crianza y el desarrollo de sus hijos. Los resultados preliminares indican que el desarrollo personal de las mujeres madres que deciden comenzar o retomar sus estudios, no sólo influye positivamente en su futuro profesional y laboral, sino en su presente como madres, acercándolas a sus hijos y a las instituciones escolares donde asisten desde otro posicionamiento personal.

Palabras clave: representaciones sociales - maternidad - universidad - inclusión

\section{ABSTRACT}

Mothers and Sons: Two developmental stages, benefitted by University Inclusion

This study explores the social representations about maternity and university students' roles, in university students who are mothers, in José C. Paz National University, located in Buenos Aires 


\section{MADRES E HIJOS: DOS ETAPAS EVOLUTIVAS BENEFICIADAS POR LA INCLUSIÓN UNIVERSITARIA}

Province, Argentina. The aim of this project is to analyze the influence of those representations on the permanence of the students - mothers in university. The methods to collect data are: semi-structured interview and free association words technique. It is expected that the data about the group studied will be useful to design inclusion strategies in function of the particular vulnerability of these women. Educative inclusion is defined by UNESCO as the process to identify and respond to the diversity of necessities of every student. To think about full university inclusion of students- mothers implies modifications in the determinant structures of university mechanisms, strategies and structural changes. Social representations explored in this investigation allow, not only to identify students' needs, that influence in a substantial way in their academic integration, but also in their raising styles and their sons' development. Preliminary results show that personal development experienced by these mothers who decide to study at university, not only influence positively their professional future, but also their motherhood present, making feel them closer to their sons and feeling different in front of their sons' schools teachers and leaders.

Key words: social representations - maternity - university - inclusion

\section{ANTECEDENTES}

Existen diversos antecedentes en el campo de las investigaciones sobre RRSS de la maternidad en estudiantes. En primer lugar se encuentran aquellas que estudian la maternidad en adolescentes de escuela secundaria, evidenciando una relación directa entre embarazo, maternidad y deserción escolar o postergación de los estudios (Gómez Sotelo, 2012). Los resultados del trabajo de Gómez Sotelo muestran que las representaciones sociales del embarazo y la maternidad en adolescentes, están asociadas a: nuevo estatus social, miedo al rechazo familiar y decisiones trascendentales.

En el ámbito académico, tanto a nivel nacional como internacional, se encuentran investigaciones que indagan sobre las representaciones que poseen los alumnos universitarios sobre distintos aspectos de la maternidad y los estudios universitarios.

A nivel internacional, Sandoval y Sepúlveda (2014) Ilevaron adelante un trabajo acerca del ser madre y estudiante universitaria en la Universidad de Santiago de Chile, teniendo como objetivo identificar las implicancias psicosociales de ser madre en estudiantes universitarias. Los autores concluyen que aunque es complejo conllevar el rol de madre y estudiante a la vez, puede haber compatibilidad entre ambos, pero a su vez encontraron dificultades que se presentan a la madre al estar estudiando en la universidad.

Lamus Canavate (1999), llevó a cabo una investigación acerca de las RRSS sobre maternidad y paternidad en cinco ciudades colombianas. Algunos de los interrogantes que se plantea en su trabajo son de sumo interés para este proyecto: cómo influyen los nuevos roles de la mujer en la forma que asume su maternidad, y cuáles son las paradojas y contradicciones para la mujer en esta tarea. Uno de los objetivos de dicha investigación ha sido conocer cómo inciden esos cambios en relación con la nueva función proveedora de la mujer; el mejoramiento del nivel educativo, y su vinculación al espacio público. Esos tres ámbitos de desarrollo habían sido históricamente ocupados por hombres, mientras la mujer ejercía el rol de crianza y cuidado de la casa, quedando relegada a un espacio reducido a la vida privada, que limitaba su vinculación con otras esferas de desarrollo personal, como la académica.

Otra investigación colombiana acerca de las representaciones sobre paternidad y maternidad, postula tendencias cambiantes desde un modelo tradicional hacia otro de transición y ruptura respecto de estos roles. Este estudio indaga cómo otros proyectos de vida se suman al de la maternidad generando ambigüedad en las mujeres, ya que estos nuevos proyectos "resquebrajarían" las RRSS que asimilan el "ser mujer" con la maternidad, (Puyana Villamizar y Mosquera Rosero, 2005). 
Las autoras concluyen que las nuevas representaciones de "ruptura", tales como la de madre proveedora, generan la desaparición de la desigualdad de los sexos en el plano económico pero no cultural. Las mujeres cuyas RRSS se corresponden con las de la tendencia de transición y ruptura "experimentaron sentimientos contradictorios ante la llegada de la prole debido a que cursaban carreras profesionales o actividades laborales que les generaban bienestar y que chocaban con las demandas propias de la maternidad. De la misma manera, las contradicciones y las culpas estuvieron presentes cuando advirtieron que no contaban con todo el tiempo para dedicarse a la crianza de la progenie", (Puyana Villamizar y Mosquera Rosero, 2005, p.19).

En la Argentina, Colombo y otros (2010), abordaron las RRSS sobre la maternidad y paternidad en adolescentes tardíos universitarios sin hijos (Colombo y otros, 2010). Dentro de las conclusiones, resulta interesante que respecto del lugar que ocupa la paternidad/maternidad en el proyecto vital, los adolescentes pensarían en ello cuando finalicen los estudios, cuando tengan los recursos para satisfacer todas sus necesidades, cuando tengan una pareja estable, cuando tengan estabilidad afectiva; etc.

En la investigación que aquí se presenta es de suma importancia tomar en cuenta los datos reseñados, para estudiar las representaciones sociales (en adelante: RRSS) de las alumnas universitarias que sí tienen hijos. Se asume entonces que las RRSS sobre la maternidad, el rol de estudiante, el empleo, etc., son datos relevantes para comprender en qué condiciones se encuentran las mujeres-madres que cursan estudios universitarios.

Objetivos

Si se asume que la cuestión de las alumnas madres es relevante en la agenda educativa universitaria, debe considerarse necesario:

Identificar aquellos factores que obturan su plena inclusión académica

Reconocer las necesidades de las estudiantes, sus aspectos más vulnerables, para así poder reducir su exclusión del sistema educativo universitario.

Se propone entonces indagar acerca de las RRSS sobre la maternidad; el rol de alumna universitaria y el empleo, como realidades coexistentes, en las alumnas de la UNPAZ. Conocer las RRSS circulantes es trascendental para responder a las necesidades de las alumnas - madres desde la institución.

Es por ello que la pertinencia del proyecto radica en el siguiente interrogante: ¿De qué manera la universidad puede tomar en cuenta las RRSS acerca de los roles de alumna; madre; y trabajadora de su alumnado femenino, para generar estrategias que favorezcan la inclusión de las alumnas madres en su propio seno?

\section{MUESTRA}

La presente investigación, de carácter exploratorio y descriptivo, analítico y relacional, emplea una metodología de carácter cualitativo y se lleva a cabo con una muestra intencionada.

Población: Alumnas de la Universidad Nacional de José. C. Paz (en adelante: UNPAZ) en ejercicio del rol materno, primera generación de estudiantes de grado en su familia de origen, pertenecientes a sectores populares de bajos recursos.

Muestra: se utilizará un muestreo intencionado, a través de la selección de alumnas-madres, en condición de regulares de $1^{\circ} 02^{\circ}$ año de la carrera de Trabajo Social de la UNPAZ.

\section{METODOLOGíA}

Para llevar adelante el estudio exploratorio que se presenta, se aplican métodos de recolección de datos cualitativos. Los métodos a emplear son: la entrevista semi-dirigida y la asociación de palabras. 


\section{Instrumentos de recolección de datos:}

Entrevistas semi estructuradas: Las entrevistas se llevarán a cabo individualmente siguiendo el método clínico (Reuchlin, 1971). El entrevistado responde a los ítems sobre los que el entrevistador interroga, pero con respuestas abiertas, y al mismo tiempo son retomadas para repreguntar. Las mismas serán grabadas en audio y una vez realizadas, se transcriben para un mejor empleo de los datos. Este tipo de entrevista se considera la vía regia para acceder al pensamiento espontáneo del sujeto, pues permite un interjuego dialéctico entre hipótesis previas del investigador y datos empíricos que las reorientan durante la propia entrevista.

Técnica de Asociación de Palabras / Test de Asociación Libre de Palabras (TALP): El TALP es caracterizado por ser una técnica proyectiva que favorece a los individuos a revelar el contenido latente de la memoria en relación a un objeto, fenómeno o situación determinado. Se pronuncian delante del sujeto palabras denominadas inductoras. En respuesta, el sujeto debe verbalizar lo más rápido posible las primeras palabras que le vienen a la mente. Luego, se crea un diccionario que incluye todo el repertorio de respuestas evocadas, que se organizan para elaborar categorías que dan cuenta de una forma de pensar y sentir en relación a la temática indagada, aportando datos para conocer las RRSS circulantes en la muestra.

\section{Resultados preliminares:}

Los datos que arrojan las entrevistas analizadas hasta el momento, han permitido un análisis preliminar que se ha desarrollado estableciendo cuatro dimensiones de análisis:

1. La relación entre la motivación a estudiar y la maternidad

2. El cambio en el modo de pensar y reflexionar como mujeres y madres a partir de sus estudios universitarios

3. La posibilidad de compatibilizar o no los roles de madre y estudiante

4. El cambio en el vínculo madre - hijo que acarrea su inserción en la vida universitaria

Tales dimensiones permiten organizar la información recolectada, para dar paso a la interpretación de los datos en función del marco teórico seleccionado: la teoría de las Representaciones Sociales de Moscovici, S. (1984).

El análisis de las primeras entrevistas muestra la importancia que tienen los estudios universitarios no sólo como proyecto personal de las alumnas - madres, sino para el modelo que ofrecen a sus hijos, diferente al que recibieron ellas, siendo en su totalidad primera generación de estudiantes universitarias en sus familias. En ese sentido, Ana Laura, 21 años, habla acerca de la relación entre la motivación a estudiar y la maternidad: "Si, siempre un futuro para mi hijo, (...) para que se identifique en mi y que el día de mañana decida estudiar (...) yo siento que soy la referente, que estudie, que sea alguien en la vida." Frente a la misma pregunta, Johana, 36 años, comenta: "sí, hay una relación (...) yo siendo mamá, quiero ser universitaria para que el día de mañana mis hijos puedan tener el mismo ejemplo, entonces... hoy decidí estudiar...". En este mismo sentido, Gladys, 45 años, ante la pregunta por el impacto de estar estudiando en su rol de madre responde: "un impacto pero positivo, de hecho lo veo todo los días, mi hija apenas llega de jardín me pregunta: hoy tenés facu? Es lo primero que me dice y también me dice: cuando yo sea grande también voy a ir a la facu, entonces es como que ella mira mi ejemplo y lo incorpora...".

Respecto a la relación entre estudiar y ser madre, compatibilizando ambos roles, Sabrina sostiene: "se compatibilizan bien porque uno lleva la universidad al hijo y el hijo a la universidad, (...) él tiene 14 años y ya está haciendo cuestionamientos a bajadas de línea de la sociedad, las relaciones sociales que están pautadas como las propagandas (publicidades), entonces (...) yo lo estoy Ilevando a la universidad con sus 14 años, (...), o cosas que dice, explicarle por qué están mal y 
que no tiene que seguir repitiendo lo que los demás dicen...". Frente a la misma pregunta, Carolina (28 años) responde: "sí, me cambia mucho, por ejemplo el conocimiento que he obtenido a través de las materias, (asignaturas) cambiaron mi forma de actuar ante mis hijas". Así, la posibilidad de estudiar modifica los vínculos entre madres e hijos, por el grado de conceptualización que las madres logran sobre el proceso de crianza, y también por el efecto menos pensado de compartir roles, madres e hijos, ambos estudiantes, unidos en el proceso de aprendizaje, como explica Laura (40 años): "Sí, me motiva a que yo puedo ayudar a mi hijo de doce años en cosas que no sabía (...) cuando llego a mi casa (...) le digo que es re lindo (estudiar) y que cuando él sea grande también va a aprender un montón de cosas. Yo me pongo a estudiar y él se pone conmigo, me ayuda, me toma como una lección".

El cambio que manifiestan a partir de su experiencia universitaria impacta en la forma de entender a sus hijos y explicarles las pautas de crianza propias o escolares así como los contenidos de los medios de comunicación. Se trata de mujeres adultas, que han decidido por convicción propia encarar un proyecto educativo - profesional dentro del sistema universitario. Las frases de las entrevistadas dan cuenta de una valoración sumamente positiva acerca de su inserción universitaria, dados los efectos de la misma no sólo en su futuro, sino en el de sus hijos a través del proyecto identificatorio. En ese sentido, Antonia (37 años), manifiesta: “... ser estudiante universitaria es una buena elección a futuro, aparte es un buen ejemplo para los hijos. Cuando me siento a leer en casa, mis hijos van y vienen y agarran una hoja y me dicen: a ver qué lees! Todo el tiempo me preguntan cosas de acá, es más, también han venido a cursar varias veces porque querían ver cómo es una universidad". Surge aquí una idea nueva, la de integrar los hijos a la universidad. Lorena, (34 años), Io plantea como necesidad: "es como que hay un quiebre entre la maternidad y la universidad (...) a veces siento que ingresando a este mundo tengo que dejar el otro (...) no hay lugar en este mundo para ellos, y a mí me encantaría que las dos cosas se puedan unir, no solo el hecho de traer a mis hijos a la universidad, si bien hay profesores que me lo permiten, siento que no es el lugar de ellos (...) no está preparado para recibirlos, y eso tiene que estar considerado porque somos muchas más las que somos madres que las que no (...) me gustaría que ella diga "qué lindo donde va mi mamá",(...), y la verdad es que a mí no solo me genera incomodidad traerla sino que yo siento que a ella también,(...) debería haber algo como para que ellos se sientan incentivados y digan yo quiero ir ahí donde va mi mamá, yo fui, me sentí cómoda, la pasé bien y el día de mañana quiero ir".

\section{DISCUSIÓN:}

Durante mucho tiempo, la centralización de las universidades públicas en las principales ciudades de nuestro país, las ha poblado de alumnos que tienen el acceso facilitado, (por cercanía o posibilidades económicas), excluyendo a quienes no pueden trasladarse hasta ellas. En esas universidades tradicionales, existe una postergación del proyecto de paternidad/maternidad en pos de terminar los estudios, (Colombo y González, 2010). Es por ello, que en la Ciudad de Buenos Aires se registra la menor tasa de fecundidad adolescente (Pantelides y Binstock 207:28; 2001). Pero la Argentina ha vivido en la última década una transformación notable en su oferta de formación académica, lo que ha permitido a miles de ciudadanos el acceso a una formación de grado. Así, llegan a la universidad una gran cantidad de alumnas-madres (adolescentes, jóvenes embarazadas y mujeres con hijos pequeños) de sectores populares, dispuestas a continuar su desarrollo profesional en simultaneidad con la maternidad. De aquí surge la importancia que tienen las RRSS de las alumnas acerca de la maternidad y los estudios de grado para todos los actores implicados en su trayectoria universitaria. Estos datos tendrán suma relevancia para el diseño de políticas universitarias, estra- 


\section{MADRES E HIJOS: DOS ETAPAS EVOLUTIVAS BENEFICIADAS POR LA INCLUSIÓN UNIVERSITARIA}

tegias docentes, cursos de extensión universitaria, guarderías universitarias, ludotecas multi-edad, etc.

Es a través de los procesos de objetivación y anclaje, que las RRSS impactan en el contexto social. La objetivación le da forma a la imagen y la estructura, esto es: permite la construcción de significados que dan sentido a la existencia, (Jodelet, 1986). Un denominador común en las entrevistadas es la imagen de "universitario" como portadora del significado de "ser alguien en la vida", "estar orgulloso", "dar el ejemplo". Por su parte, el anclaje, hace alusión al "arraigamiento" social de la representación y al papel que ésta juega al dinamizar la vida social, (Jodelet, ob. cit). En este sentido, la vida universitaria estaría dinamizada por el arraigamiento de la representación de las estudiantes como alumnas y no como madres, anclando en el ámbito universitario el quiebre entre los mundos donde ejercen ambos roles.

Por otro lado, para Jodelet (1991), las RRSS refieren a una forma de conocimiento, elaborada y compartida socialmente, que tiene un objetivo práctico y contribuye a la construcción de una realidad compartida por un grupo social, por lo que son dinámicas y cambiantes. Es precisamente por el sentido cultural y dinámicamente cambiante, que el "deber ser" de una madre, ya no coincide necesariamente con el formato de familia occidental, moderna y de clase media (Castells, 1998). Hay muchas formas de "ser madre", y entre ellas, existe la de complementar ese rol con el de estudiante y sostén de hogar, lo que no coincide con el estereotipo de la buena madre, descripta como un ser abnegado que posterga sus proyectos personales por el "bien" del niño. Tradicionalmente la madre debía quedarse en casa, evitando así una actitud abandónica dejando al niño a su buena suerte. Así, "alejarse de aquel ideal de madre incondicional, madura y preparada para la función asignada, convierte en foco de sospecha a las mujeres, sobre todo si se trata de jóvenes, pobres y solteras", (Marcus, J. 2006, p.104). En los últimos tiempos, parecieran coexistir el modelo patriarcal tradicional (en el cual el rol de la mujer está directa y casi únicamente ligado a la maternidad, las tareas domésticas y de crianza), con otro modelo en el que las mujeres combinan la maternidad con otros proyectos de vida. Por ello, es válido preguntarse: ¿Cuál es el "bien del niño"? ¿Es la postergación de los proyectos de las mujeres en función de la crianza, necesariamente un bien para sus hijos? ¿0 puede por el contrario, la combinación de la maternidad con otros proyectos, tener impacto positivo en los niños que esas madres crían? En paralelo a este eje de la discusión, surge otro no menos polémico, pues si el primero pone en duda el modelo patriarcal tradicional, el segundo hará lo propio con el modelo universitario tradicional: $¿$ “Deben" las universidades contemplar estrategias que "incluyan a los hijos de las alumnas madres" como variable a tener en cuenta en sus reglamentos, estructuras, dispositivos, etc.; para que ellas puedan concluir sus estudios? Ese no ha sido uno de los idearios de los tradicionales centros del saber, donde se oscila entre el modelo profesional y el investigativo, pero en todos los casos, siendo instituciones sociales históricamente elitistas.

La historia de la Argentina da cuenta de una apertura ininterrumpida de la universidad para convertirse en una institución educativa de masas. Así, las universidades dejaron de ser centros de formación de la elite aristocrática para pasar a ser proveedoras de títulos habilitantes cuyo ejercicio permitía el ascenso social, (Buchbinder y Marquina, 2008). El proceso por el cual la universidad argentina se ha diferenciado del modelo universitario tradicional se ha dado en dos tiempos: primero a través de la educación universitaria pública y gratuita de ingreso irrestricto, y más recientemente a través de la creación de universidades nacionales emplazadas en zonas periféricas del país facilitando el acceso sectores de la población que de otro modo no continuarían sus estudios finalizada la escolarización.

La universidad no ha sido la única institución social que ha cambiado, también la familia: “... hombres y mujeres han construido nuevos sentidos a su existencia en torno a la forma de ser padres 
o madres. Por un lado, se ha resquebrajado la ecuación mujer igual madre a través de la cual se le había asignado a la mujer la maternidad como proyecto central de la vida. Por otra parte, también se está debilitando la figura del padre proveedor, ajeno a la expresión de los afectos y centro de autoridad de la familia", (Puyana Villamizar y Mosquera Rosero, 2005, p.18).

Esos nuevos sentidos circulantes estarían impactando en la toma de decisiones de las mujeres a la hora de planificar su proyecto de vida. Es así, que la maternidad y el acceso a los estudios universitarios de nuevos sectores sociales, conduce a la necesaria complementariedad de los roles de madre y alumna. Esto obliga a toda universidad que pretenda ser inclusiva, a tener en cuenta dicha complementariedad como una situación vital frecuente de su estudiantado, que modifica la estructura universitaria tradicional.

\section{CONCLUSIONES:}

Las alumnas-madres son las protagonistas de un nuevo capítulo en la historia universitaria argentina, dado que intentan ejercer estos dos roles que, históricamente, no han sido compatibles, ya que la representación del alumno siempre ha sido relacionada, con la del niño, asumiendo ambas actividades: educativas y parentales, como excluyentes.

La docilidad, inmadurez y falta de experticia propia del niño fueron características trasladadas al alumno sin importar su edad (Baquero y Terigi, 1996). La naturalización de esta idea también podría ser, entre otras, una de las razones por las que la posición de Alumna y Madre son difíciles de conjugar, si un alumno es reducido a la posición de niño: ¿Cómo puede una niña ser madre? Así, las RRSS de alumno, y de madre se autoexcluyen en el ámbito académico desde una perspectiva tradicional y desactualizada del alumno, que no contempla el cambio acaecido en los roles sociales que el sujeto de aprendizaje lleva adelante en simultáneo a sus estudios en la actualidad. Es por ello, que la investigación pretende aportar datos para una mirada más cercana a las realidades e intereses actuales de gran parte del alumnado, explorando cómo piensan y sienten respecto de los distintos roles que asumen mientras son parte de la universidad.

Para González Rey (2003), el rol de las representaciones y prácticas sociales es mediar en la compleja relación entre las entidades individuales y sociales, siendo la universidad una entidad social compuesta por múltiples actores individuales. En el alumnado de la UNPAZ la presencia de estudiantes de sexo femenino, madres o embarazadas, es notable. Muchas son además madres trabajadoras, en algunos casos sostenes de hogar, con empleos precarios. Por este motivo, resulta relevante conocer cuáles son las RRSS de esas alumnas acerca de las posibilidades y dificultades que encuentran a la hora de cumplir con ambos roles sociales: el de madres y el de estudiantes universitarias.

La inclusión según la Real Academia Española es la "acción y efecto de incluir", el hecho de ser una "acción" la constituye una tarea activa y en constante movimiento para poder llegar al "efecto" esperable de incluir a alguien en un lugar determinado. Pensar la Inclusión en las instituciones educativas, conlleva el hecho de ampliar el término a Inclusión Educativa. El concepto de inclusión educativa (UNESCO 2008) plantea la idea de que niñas, niños y jóvenes tienen derecho a una educación que brinde equivalentes oportunidades de aprendizaje en diferentes tipos de instituciones sin que ello dependa de sus antecedentes sociales y culturales e independientemente de sus diferencias en las habilidades y capacidades. Se debe considerar a la Universidad como una institución educativa más, que dentro de un proyecto de universalización del acceso a estudios universitarios y terciarios llevado a cabo en nuestro país en la última década, no es ajena a la necesidad de pensarla desde políticas de inclusión educativa.

Las políticas de Inclusión Educativa se plantean cómo promover e implementar el derecho a la 
educación como oportunidades y logros concretos, factibles y perdurables para que todos puedan crecer social e individualmente y tener una oportunidad real de éxito en la vida. En la $48^{\circ}$ conferencia sobre Educación de la UNESCO, se declara la necesidad de considerar que el derecho a la educación contempla y garantiza el acceso de niños y adolescentes, con especial énfasis en las jóvenes embarazadas y madres adolescentes, inmigrantes, poblaciones autóctonas, minorías y personas con capacidades diferentes. Por esta razón en nuestro país es necesario comenzar a dar lugar a la situación particular de las alumnas/madres y estudiantes universitarias. Rosa María Torres (2006) destaca “...que el derecho a la educación supone el desarrollo de una educación en sintonía con el tiempo, las realidades y las necesidades de aprendizaje de las personas en cada contexto y momento", (Opperti, 2008, p. 3).

Ahora bien, para indagar acerca de las RRSS sobre la maternidad, así como del "rol de alumno universitario", como variables involucradas en el proceso de inclusión educativa de las madres, es necesario considerar los aspectos conceptuales que se han ido definiendo para esas dos figuras sociales a lo largo del tiempo. Para ello debe tenerse en cuenta que en ambos casos se trata de constructos culturales, que varían no sólo de acuerdo al tiempo y lugar en que se analizan sino también de acuerdo a la clase social de pertenencia.

Se asume entonces, que no existe una sola forma de ser madre, ni de ser universitario. Ambas son construcciones socio-culturales que varían según el contexto y no pueden definirse de forma determinante ni por cuestiones instintivas ligadas el género femenino en un caso, ni por descendencia de generaciones de universitarios en el segundo.

En los resultados obtenidos hasta el momento, el ejercicio de ambos roles en simultáneo pareciera tener retroalimentación positiva: la maternidad brinda motivación para que las mujeres inicien y concluyan sus estudios universitarios. Asimismo, su rol de estudiantes repercute positivamente en el ejercicio de su maternidad beneficiando no sólo a su generación sino a su descendencia, proveyendo un modelo identificatorio positivo a sus hijos y ejerciendo prácticas de crianza más reflexivas. Es así, que ambas etapas evolutivas se verán beneficiadas si se desarrollan estrategias destinadas a la inclusión y permanencia de las alumnas - madres en el ámbito universitario.

\section{REFERENCIAS BIBLIOGRÁFICAS:}

Aguayo, F. (2003). Paternidades, políticas y programas. Revisión de acciones y medidas para promover la participación del padre en el cuidado y la crianza de sus hijos e hijas. Santiago: UNICEF.

Anguena, M. y otros: Métodos de investigación en psicología. Síntesis Psicológica. Madrid. España.1998.

Arango, L. G., León, M., Viveros, M. (Comp.) (2000). Género e identidad. Ensayos sobre lo femenino y lo masculino. Madrid: TM.

Aries, P. y Dubby, G. (1989) Historia de la vida privada. Altea Taurus, Alfaguara. Madrid.

Avendaño, C., Krause, M. \& Winkler, I. (1993). Representaciones sociales y teorías subjetivas: Relevancia teórica y aplicaciones empíricas. Psykhe, 2(1), 107-114.

Banister, P.; Burman, E.; Parker, I.; Taylor, M. \& Tindall, C. (2004) Métodos cualitativos en psicología. México: Universidad de Guadalajara.

Baquero,R y Terigi, F ( 1996). En búsqueda de una unidad de análisis del aprendizaje escolar. Apuntes Pedagógicos, N 2.

Bauman, Z. (2001). La posmodernidad y sus descontentos. Madrid: Akal.

Berger, P. y Luckmann, T. (1995). La construcción social de la realidad. Buenos Aires: Amorrortu

Bleichmar, E. D. (1997). La sexualidad femenina. Barcelona: Paidós. 
Beauvoir, S. de (1977) El Segundo Sexo. La experiencia vivida. Ed. Siglo XX. Buenos Aires. Brown, G. y Yule, G. (1993). Análisis del Discurso. Madrid: Madrid.

Brünner, J. (1998). Globalización cultural y posmodernidad. Santiago: Fondo de Cultura Económica. Buchbinder, P. y Marquina, M. (2008). Masividad, fragmentación y heterogeneidad: el sistema universitario argentino 1983-2008, UNGS y Biblioteca Nacional, pp. 11-35.

Burin, M, y Meler, I. (1998). Género y familia. Buenos Aires: Paidós.

Canto y Rodríguez, J. E. (1998). Autoeficacia y educación. Educación y ciencia. Vol.2 №4. (P. 45 53).

Casullo, M. M. y otros (2006). Proyecto de vida y Decisión vocacional. Buenos Aires: Paidós.

Lamus Canavate, D. (1999) Representaciones Sociales de Maternidad y Paternidad en Cinco Ciudades Colombianas. Reflexión Política [online] , 1 (2) : [Fecha de consulta: 12 de julio de 2015] Disponible en:<http://www.redalyc.org/articulo.oa?id=11010211> ISSN 0124-0781

Cárdenas, M.; Parra, L.; Piccon, J.; Pineda, H. y Rojas, R. (2007). Las representaciones sociales de la política y la democracia. Última Década, 26, 55-80. Viña del Mar, Chile.

Castorina, J.A. (2005). La investigación psicológica de los conocimientos sociales. Los desafíos a la tradición constructivista. En J. A. Castorina (Coord.). Construcción conceptual y representaciones sociales. El conocimiento de la sociedad (pp. 19-44). Buenos Aires: Miño y Dávila.

Colombo, M.E. y Gonzales, A. (2010). Análisis de resultados de entrevistas a Jóvenes universitarios sin hijos sobre representaciones sociales de maternidad y paternidad. II Congreso Internacional de Investigación y Práctica Profesional en Psicología XVII Jornadas de Investigación Sexto Encuentro de Investigadores en Psicología del MERCOSUR. Acta Académica. 260-261. Facultad de Psicología - Universidad de Buenos Aires, Buenos Aires.

Cordero, L.; Robles, C. (2012). Representaciones sociales del concepto 'familia' en estudiantes de T. Social. (UnLaM). INFORME FINAL: Código: A 137. Unidad Ejecutora: Departamento de Humanidades y Ciencias Sociales; Carrera de Trabajo Social. La Matanza, Provincia de Buenos Aires.

Duschatzky, S.; Corea, C. ( 2001).Chicos en banda: Ios caminos de la subjetividad en declive de las instituciones. Buenos Aires. Paidós.

Feldman, G.; González, A.; D’Avirro, M. J.; Iglesias, S.; Colombo, M. E. (2009). Análisis de resultados de entrevistas a jóvenes universitarios sin hijos sobre representaciones sociales de maternidad y paternidad. Congreso Internacional de Investigación de la facultad de Psicología de la Universidad Nacional de La Plata. Buenos Aires, Argentina.

Fuller, N. (Ed.) (2000). Paternidades en América Latina. Lima: Pontificia Universidad Católica del Perú. Fondo Editorial.

Gergen, K. (1997). El yo saturado. Buenos Aires: Paidós.

Glaser, G. \& Straus (1967). The discovery of the grounded theory: strategies for qualitative research. New York: Aldine Publishing Company.

Gomez Sotelo, A. (2012). Representaciones sociales del embarazo y maternidad en adolescentes primigestantes y multigestantes en Bogotá. Salud Pública, 14, 189-199.

Gomez-Sotelo, A.; Gutierrez-Malaver, M.; Izzedin-Bouquet, L. ( 2011). Representaciones sociales del embarazo y la maternidad en adolescentes primigestantes y multigestantes en Bogotá. Revista de salud Pública ISSN 0124-0064

González Rey, F. B. (2003). Democracy and citizenship: an analysis involving social representations and social subjectivity. En M. Lavallée, S. Vincent, C. Ouellet \& C. Garnier (Eds.). Les représentations sociales. Constructions nouvelles (pp: 217-229). Montréal, Canada: GEIRSO - Université du Québec á Montréal. 
González, M. (1991). La paternidad humana y los aportes específicos del padre y la madre en la educación de los hijos. Pontificia Universidad Católica de Chile, Santiago.

Hernando, A. (2000). Factores estructurales asociados a la identidad de género femenina. La no-inocencia de una construcción socio-cultural. En Hernando, A. (Ed.), La construcción de la subjetividad femenina. Madrid: Instituto de Investigaciones Feminista de la Universidad Complutense de Madrid.

Jiménez, A. B. (2004). La paternidad en entredicho. Gazeta de Antropología, 20, Artículo 21. Extraído 10 de Septiembre, 2005, de http://www.ugr.es/ pwlac/G20-19AnaBelen-Jimenez-Godoy.html

Jodelet, D. (1986). La representación social: fenómenos, conceptos y teoría. En S. Moscovici: Psicología social II. Pensamiento y vida social, psicología social y problemas sociales. Barcelona: Paidós.

Jodelet, D. (Ed.) (1991). Les représentations sociales. París: PUF.

Marcus, J. (2006). Ser madre en los sectores populares: una aproximación al sentido que las mujeres le otorgan a la maternidad. Revista Argentina de Sociología. Buenos Aires; p. 99 - 99. ISSN: 1667-9261

Milicic, N., Alcalay, L. y Torretti, A. (1992). Diseño de un programa para favorecer la identidad femenina en alumnas de $7^{0}$ y $8^{0}$ año de educación general básica (Proyecto FONDECYT 1992/0799). Santiago: Pontificia Universidad Católica de Chile, Escuela de Psicología

Moscovici, S. (1979). El psicoanálisis, su imagen y su público. Buenos Aires: Huemul.

Moscovici, S. (1984). Psicología Social, I y II, Barcelona: Paidós.

Moscovici, S. (2003). La conciencia social y su historia. En Castorina, A. (Comp.), Representaciones sociales. Barcelona: Gedisa.

Opertti, R. (2008). Inclusión Educativa: El Camino del Futuro Un desafío para compartir. 48ª reunión de la Conferencia Internacional de Educación

Pantelides, E. y Binstock, G. (2005). La Fecundidad Adolescente Hoy: Diagnostico Sociodemografico, en Gogña, Monica (coord.) Embarazo y maternidad en la adolescencia. Estereotipos, evidencias y propuestas para políticas públicas, Buenos Aires, UNICEF, CEDES, pp. 77-112.

Puyana Villamizar, Y. y Mosquera R. C. (2005). Traer "hijos o hijas al mundo": significados culturales de la paternidad y la maternidad. Revista Latinoamericana de Ciencias Sociales, Niñez y Juventud, 3(2), 111-140. Visitado Julio 12, 2015, desde http://www.scielo.org.co/scielo.php?script=sci_arttext\&pid=S1692$715 X 2005000200005 \& \operatorname{lng}=e n \& t \operatorname{lng}=e s$. .

Reuchlin, M. (1971). Les méthodes en Psychologíe. Paris: PUF

Sandoval, J.; Spúlveda, B.; Amaro, R.; Jara, M. y Vergara, J. (2014). Ser madre y estudiante universitaria en la Universidad de Santiago de Chile: un estudio exploratorio acerca de las implicancias psicosociales en el enfrentamiento de ambos roles. (Metodología de Investigación Cualitativa II Escuela de Psicología Universidad de Santiago de Chile). 2014. Volumen 1, Número 1. Págs. 23 - 39. Santiago de Chile. Revista de Estudios Cualitativos.

Sotelo Gómez, A. (2012). Representaciones sociales del embarazo y maternidad en adolescentes primigestantes y multigestantes en Bogotá. Salud Pública, 14, 189-199.)

Torres, M. R. (2006). Derecho a la educación es más que el acceso a la escuela. EL derecho a la Educación en un mundo globalizado. Volumen I (coordinado por L. M. Naya y P. Dávila). Donostia: Espacio Universitario Erein.

Viveros, Mara. La Problemática de la representación social y su utilidad en los estudios de salud y enfermedad. En: Revista el Boletín. 
Wagner, W. y Elejabarrieta, F. (1994). Representaciones sociales. En F. Morales (Coord.), Psicología social. Madrid: McGraw-Hill. Castorina, J.A. (2005). La investigación psicológica de los conocimientos sociales. Los desafíos a la tradición constructivista. En J. A. Castorina (Coord.). Construcción conceptual y representaciones sociales. El conocimiento de la sociedad (pp. 1944). Buenos Aires: Miño y Dávila.

Zubieta, E. (1997). La psicología social y el estudio de las representaciones sociales. Revista del Instituto de Investigaciones de la Facultad de Psicología UBA, 2 (2), 119- 136. Buenos Aires. 
